Recebido em 11/2011. Aceito para publicação em 03/2012.

\title{
CARACTERÍSTICAS DO ATENDIMENTO AOS PACIENTES COM DOR PRECORDIAL NO PRONTO ATENDIMENTO DE UM HOSPITAL GERAL
}

\section{SERVICE OF PATIENTS WITH CHEST PAIN IN THE EMERGENCY ROOM OF A GENERAL HOSPITAL}

\author{
Selma de Lima Faria ${ }^{1}$ \\ Regimar Carla Machado ${ }^{2}$ \\ Carolina Vivian Gianvecchio ${ }^{3}$
}

RESUMO: A I Diretriz da Dor Torácica de 2002 recomenda que o paciente com suspeita de Síndrome Coronariana Aguda (SCA) na sala de emergência deva ser submetido a um eletrocardiograma em 5 a 10 minutos. Esta pesquisa teve como objetivos levantar o tempo de evolução da dor precordial do paciente atendido no pronto atendimento de um hospital geral e mensurar o tempo da realização do eletrocardiograma. Estudo descritivo, exploratório, retrospectivo e com uma abordagem quantitativa por meio de um protocolo para análise das fichas de pacientes com Infarto Agudo do Miocárdio (IAM). Verificou-se que dos 14 pacientes, 10 (71,4\%) eram homens, 8 (57,2\%) tinham entre 50 a 69 anos, 6 (42,9\%) não havia registro do tempo de dor precordial e 10 (71,4\%) realizaram eletrocardiograma em tempo médio de 26,6 minutos. Este estudo evidenciou a importância da implementação de um protocolo que normatize o tempo de realização de um eletrocardiograma além de treinamento específico de interpretação do citado exame aos enfermeiros que atuam em pronto atendimento.

Palavras-chave: infarto agudo do miocárdio; atendimento de emergência; enfermeiro; eletrocardiograma.

ABSTRACT: The Chest Pain Guideline from 2002 recommends that patients with suspected Acute Coronary Syndrome (ACS) in the emergency room should be submitted to an electrocardiogram in 5 to 10 minutes. This research aimed to increase the evolution time of chest pain patients in the emergency room of a general hospital and measure the time to the electrocardiogram. This descriptive, exploratory, retrospective, and quantitative approach used a protocol to analyze the records of patients with Acute Myocardial Infarction (AMI). Of 14 patients, 10 (71.4\%) were men, 8 (57.2\%) were between 50 and 69 years, 6 (42.9\%) had no record of the time of chest pain, and 10 (71.4\%) underwent electrocardiography at a mean of 26.6 minutes. This study showed the importance of implementing a protocol that will regulate the time of performing an electrocardiogram as well as specific training for interpreting that examination to nurses working in emergency care.

Keywords: acute myocardial infarction; emergency care; nurse; electrocardiogram.

\footnotetext{
1 Enfermeira Especialista em Cuidados Críticos/Cardiologia - Universidade do Vale do Paraíba - Univap. Enfermeira Supervisora na Unidade de Terapia Intensiva da Santa Casa de São José dos Campos, SP. E-mail: sel_jac@hotmail.com. ${ }^{2}$ Doutora em Ciências - Universidade Federal de São Paulo, Coordenadora e docente da Especialização de Enfermagem em Cuidados Críticos/Cardiologia - Univap. E-mail: regimarcarla@yahoo.com.br.

${ }^{3}$ Enfermeira Especialista em Cuidados Críticos/Cardiologia - Univap. Enfermeira coordenadora da Angiocenter Hemodinâmica e Angiografia Digital. E-mail: carolangio@hotmail.com.
} 


\section{INTRODUÇÃO}

O Infarto Agudo do Miocárdio (IAM) é definido como a oclusão aguda de uma artéria coronária por um trombo, geralmente em área acometida previamente por placas de aterosclerose. A relação causal entre trombose da artéria coronária e IAM foi sugerida em 1912, na descrição original da síndrome do infarto agudo do miocárdio (SILVEIRA, 2002).

O diagnóstico do IAM é realizado pelo exame clínico, que tem como sintomatologia a dor retroesternal e/ou precordial, intensa, contínua, com duração superior a 40 minutos e sem resposta adequada aos fármacos vasodilatadores, tais como os nitratos. A dor pode estar ausente em $30 \%$ a $40 \%$ dos pacientes acometidos pelo IAM. Os sintomas equivalentes a dor podem ser dispnéia, dor epigástrica, síncope, náuseas e vômitos. A resposta adrenérgica exacerbada pode ser traduzida por palidez intensa, sudorese profusa, pele fria, e cianose discreta (MARINO, 1999).

As alterações podem ser apresentadas no eletrocardiograma (ECG), em qualquer uma das fases em que se encontra 0 paciente no momento do exame. As fases são classificadas em superaguda, aguda, subaguda ou crônica. Entretanto, complementando o diagnóstico de IAM, evidencia-se a análise de exame laboratorial, como a análise dos marcadores de necrose miocárdica que permitem a confirmação do IAM, possibilitando a tomada de decisão com menor tempo de isquemia (PIEGAS et al., 2009).

Dentre os tratamentos utilizados no IAM, pode-se citar o tratamento trombolítico, a Angioplastia Transluminal Percutânea (ATC) e a cirurgia de Revascularização de Miocárdio (RM) (SILVEIRA, 2002).
Todavia, um dos desafios para reduzir a mortalidade por IAM e o grau de incapacitação gerada é melhorar o atendimento pré e intra-hospitalar. Portanto, um dos fatores responsáveis pela demora no atendimento aos cardiopatas agudos é o desconhecimento ou a negação do evento isquêmico por parte de pacientes $\mathrm{e}$ familiares, acarretando em um atraso inicial considerável para o tratamento da lesão isquêmica. Estudos demonstram que o paciente na eminência do IAM vivencia a experiência da ruptura com a vida cotidiana, provocada pelo aparecimento de dor, dispnéia e sensação de morte, por conseguinte, as mais diversas ações, nessa fase, apontam para a importância da atuação do enfermeiro nos programas educativos, conscientizando os pacientes a observarem os sinais e sintomas dos eventos cardiovasculares agudos, além de salientar a importância da procura imediata de atendimento médico (MUSSI, 2004; MULLER et al., 2008).

Dentre os fatores que retardam o início do tratamento, pode-se citar a demora na procura de serviço médico. A Diretriz da Dor Torácica, do ano 2002, recomenda ao paciente com suspeita de IAM na sala de emergência realizar um eletrocardiograma entre 5 a 10 minutos após sua chegada ao hospital (MULLER et al., 2008; BASSAN et al., 2002).

A literatura científica enfatiza que, para obter benefícios com a terapia trombolítica, deve-se administrar o fármaco até 6 horas de início da dor precordial. Sobretudo, após esse período, o declínio do benefício sobre a sobrevida alcança 1,6 vidas perdidas por 1000 pacientes, por hora de retardo no tratamento. Nas primeiras 6 horas, o declínio horário na sobrevida (2,6 por 1000), tanto que retardos no tratamento são 4 vezes 
mais danosos nas primeiras 6 horas do que nas segundas 6 horas, dentro desse período efetivo de tratamento. Portanto, a eficácia do tratamento consiste na identificação precoce dos pacientes que necessitam ser submetidos à terapia trombolítica e início imediato dessa terapia (AFILALO; ROY; EISENBERG, 2009; MARINO, 1999).

A ATC primária, sem o uso prévio de drogas trombolíticas, também chamadas de ATC direta, é realizada na vigência de IAM e tem por objetivo a recanalização mecânica de artéria lesionada, procurando o restabelecimento do fluxo coronariano normal. Qualquer procedimento que visa à reperfusão deve ser realizado de preferência nas primeiras 6 horas do início dos sintomas do IAM, até no máximo de 12 horas, ou quando houver persistência de isquemia miocárdica. Tem se desenvolvido como uma alternativa viável, sendo superior a terapia trombolítica em alguns aspectos, como modalidade mais eficaz no tratamento do IAM, especialmente em pacientes de alto risco, tais como infarto extenso, idosos, contra-indicação para terapêutica trombolítica, choque cardiogênico e pacientes com cirurgia de RM recente (SILVEIRA, 2002).

Estudos para a verificação da evolução dos resultados obtidos, após a aplicação da angioplastia primária no último quadriênio, demonstraram crescimento significativo de implante de stents, classificados como endopróteses intravasculares, inseridas nas paredes dos vasos, com a finalidade de ampliar sua luz e reduzir a taxa de oclusão do fluxo de sangue. $O$ sucesso do procedimento aumentou, o reinfarto foi reduzido, como, também, a cirurgia de emergência. Porém, a mortalidade permaneceu inalterada. Verificaram-se alguns preditores de insucesso a ATC, tais como idade avançada, sexo feminino, presença de diabetes mellitus e doença coronariana multiarterial, além do reinfarto (MATTOS et al., 2002).

Nesse contexto, este estudo visa levantar o tempo de evolução da dor precordial do paciente atendido no pronto atendimento de um hospital geral e mensurar o tempo da realização do eletrocardiograma.

\section{MÉTODO}

Trata-se de um estudo descritivo, exploratório, retrospectivo e com uma abordagem quantitativa. $\mathrm{O}$ escopo da pesquisa foi dividido em duas etapas: construção do instrumento de coleta de dados e aplicação do instrumento. O protocolo da pesquisa foi submetido ao Comitê de Ética em Pesquisa da Universidade do Vale do Paraíba, sendo aprovado sob 0 Parecer de $n^{-}$ H179/CEP2008.

A construção do instrumento foi realizada com base em uma busca sistematizada da revisão da literatura e no estudo proposto por Bassan et al. (2002). Para a revisão da literatura e facilitar a busca sistematizada nas bases de dados, utilizaram-se os descritores infarto do miocárdio, atendimento de emergência, enfermeiro e eletrocardiograma.

O estudo foi realizado em um hospital de médio porte do interior do Vale do Paraíba que atende uma clientela de pacientes clínicos, cirúrgicos e ambulatoriais, fazendo parte da rede de hospitais prestadores de serviço ao Sistema Único de Saúde, por intermédio do Estado de São Paulo. 
A amostra constituiu-se de 14 fichas de atendimento de pacientes que apresentaram diagnóstico de infarto agudo do miocárdio entre janeiro a dezembro de 2008.

Estabeleceu como critérios de inclusão os pacientes que apresentassem dor precordial, seguido do diagnóstico de infarto agudo do miocárdio, confirmado mediante a realização do exame clínico pelo médico e do ECG.

Foram excluídas as fichas de atendimento dos pacientes que não apresentavam diagnóstico médico de IAM e transferidos para outras instituições.

Em todas as fichas de atendimento selecionadas, foi aplicado o instrumento de coleta de dados, no período de abril de 2009, em datas e horários pré-estabelecidos pela direção do hospital, em respeito aos funcionários e à rotina da empresa.

Os dados coletados foram transferidos para uma planilha e tabulados com o auxílio do programa Microsoft Excel®, versão do Office 2007, e analisados sob a orientação estatística. Para a confecção das tabelas, foram utilizados números absolutos e percentagem.

Utilizou-se o teste $t$-Student, para comparar a média de uma amostra de Tempo de Espera, para o Eletrocardiograma em pacientes que apresentaram dor precordial ao chegar ao Pronto Atendimento em relação ao Tempo Ideal de espera (até $10 \mathrm{~min})$.

\section{RESULTADOS}

Investigação do atendimento perante pacientes que referem dor precordial ao chegar ao Pronto Atendimento de um Hospital Geral do Vale do Paraíba.

Quanto ao horário de abertura da ficha para o atendimento, verificou-se que 10 $(71,4 \%)$ pacientes foram admitidos no Pronto Atendimento antes das 18 horas e $4(28,6 \%)$ pacientes foram admitidos após as 18 horas.

\section{Tabela 1 - Perfil sócio demográfico da população estudada. São José dos Campos, 2011}

\begin{tabular}{lrr}
\hline \multicolumn{1}{c}{ GÊNERO } & N & \multicolumn{1}{c}{$\%$} \\
\hline Masculino & 10 & 71,4 \\
Feminino & 4 & 28,6 \\
Total & 14 & 100,0 \\
\hline IDADE (ANOS) & \multicolumn{1}{c}{ N } & \multicolumn{1}{c}{$\%$} \\
\hline 30 a 39 & 1 & 7,1 \\
40 a 49 & 2 & 14,3 \\
50 a 59 & 4 & 28,6 \\
60 a 69 & 4 & 28,6 \\
70 a 79 & 2 & 14,3 \\
80 e + & 1 & 7,1 \\
Total & 14 & 100,0 \\
\hline
\end{tabular}

A Tabela 1 demonstra que foram analisadas 14 fichas de atendimento, sendo $10(71,4 \%)$ pacientes homens e $4(28,6 \%)$ mulheres. Quanto a idade, 1 (7,1\%) apresentou entre 30 a 39 anos, 2 (14,3\%) apresentaram de 40 a 49 anos, $4(28,6 \%)$ entre 50 a 59 anos, $4(28,6 \%)$ entre 60 a 69 anos, 2 (14,3\%) entre 70 a 79 anos e 1 $(7,1 \%)$ apresentou mais de 80 anos. 


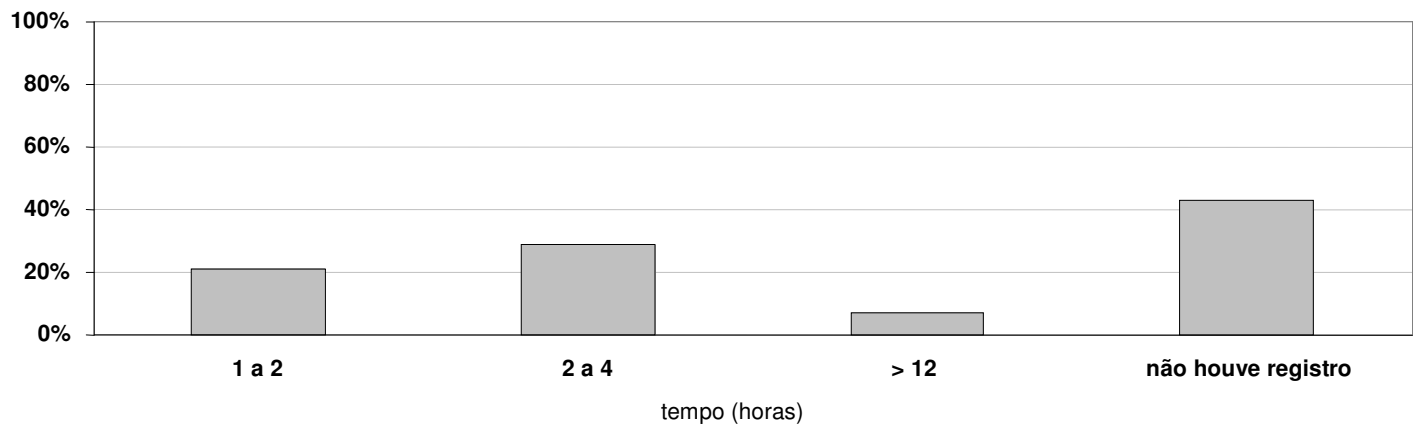

Fig. 1 - Tempo de evolução da dor precordial até o momento do atendimento. São José dos Campos, 2011.

Observa-se, na Fig. 1, que do total de $14(100 \%)$ pacientes, $3(21,4 \%)$ referiram presença de dor no tempo entre 1 e 2 horas, $4(28,6 \%)$ entre 2 e 4 horas, $1(7,1 \%)$ relatou estar com dor há mais de 12 horas e, em 6 (42,9\%) fichas de atendimento, não apresentaram registros do tempo da dor precordial.

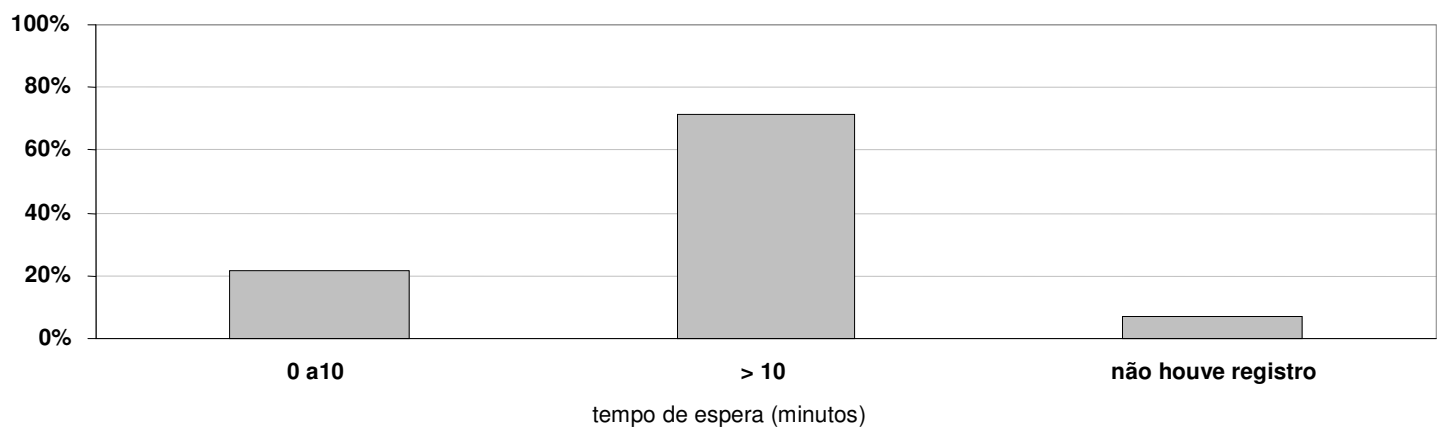

Fig. 2 - Tempo de espera para realização do eletrocardiograma a partir do momento da abertura da ficha de atendimento. São José dos Campos, 2011.

A Fig. 2 demonstra que $3(21,4 \%)$ a 10 minutos e $1(7,1 \%)$ paciente não tinha 0 tiveram o ECG realizado no tempo registro do horário de realização do exame considerado ideal e $10 \quad(71,4 \%)$ foram na ficha de atendimento.

submetidos ao ECG em um tempo superior

Tabela 2 - Estatística descritiva da variável Tempo de Espera para Realização do Eletrocardiograma. São José dos Campos, 2011

\begin{tabular}{cccccccccc}
\hline Variável & $\mathbf{N}$ & Dados & Média & DP $^{\star}$ & Mediana & Mínimo & Máximo & $\mathbf{1}^{\mathbf{0}}$ & 3 \\
\hline Tempo de Espera para & 13 & 1 & 26,6 & 21,1 & 25 & 0 & 69 & 10 & 33 \\
\hline *DP: desvio-padrão & & & & & & &
\end{tabular}


Observa-se maior tempo de espera do ideal, máximo de 10 minutos, para a paciente desde sua chegada no pronto realização do eletrocardiograma.

atendimento, quando comparada ao Tempo

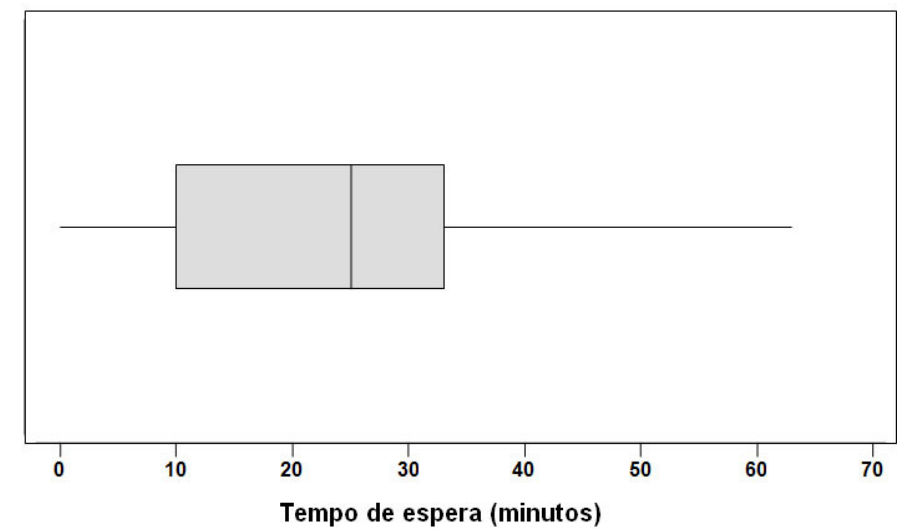

Fig. 3 - Boxplot do Tempo de Espera para Eletrocardiograma. São José dos Campos, 2011.

O gráfico Boxplot ilustra a distribuição dos tempos de espera para realização do eletrocardiograma, com o valor mínimo de zero (minutos) de espera, e o valor máximo, discrepante da amostra (outlier) igual a 69 minutos de espera, que é um valor muito maior que os comumente encontrados na amostra. A mediana dos tempos de espera é igual a 25 minutos e os tempos se encontram mais concentrados entre 10 minutos (1ำ quartil) a 33 ( $3^{\circ}$ quartil) minutos de espera para realização do eletrocardiograma.
Como o "valor p" do Teste t, para uma amostra ( $p=0,015)$, é inferior ao nível de significância do teste de 0,05 , rejeita-se a Hipótese nula que supõe a média do Tempo de espera para o Eletrocardiograma igual a 10 minutos, ou seja, existe diferença significativa na média da variável Tempo de Espera para o Eletrocardiograma dos pacientes que referem dor precordial ao chegar ao Pronto Atendimento de um Hospital Geral do Vale do Paraíba, em relação ao Tempo Ideal de 10 minutos de espera.

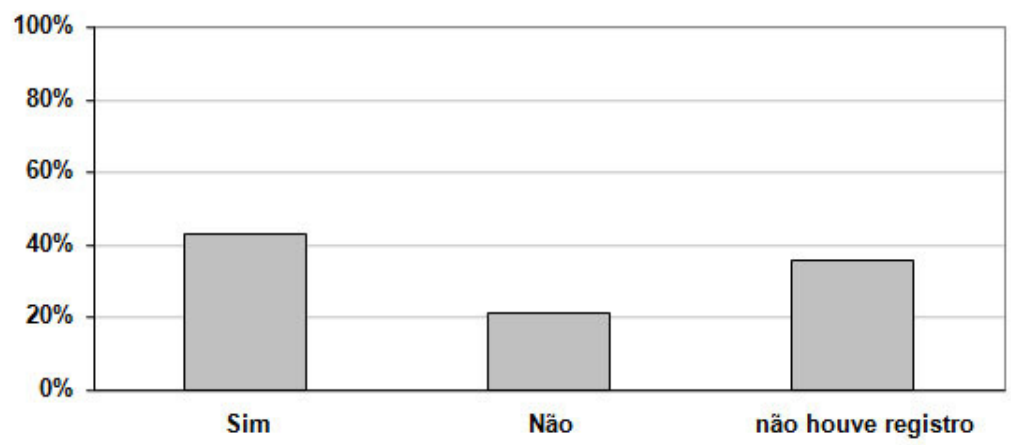

Fig. 4 - Diagnóstico de infarto agudo do miocárdio, registrado no eletrocardiograma durante o atendimento na sala de emergência. São José dos Campos, 2011. 
Observa-se que 6 (42,9\%) pacientes apresentaram alteração no traçado do eletrocardiograma, $3 \quad(21,4 \%)$ não apresentaram alteração, enquanto que em 5 $(35,7 \%)$ fichas de atendimento não há registro e/ou eletrocardiograma anexado.

\section{DISCUSSÃO}

A literatura enfatiza 0 atendimento rápido ao paciente com dor precordial na sala de emergência, salientando a prioridade da realização do exame de eletrocardiograma em até 10 minutos, para que se houver o diagnóstico do IAM, o paciente receba o tratamento adequado no menor tempo possível (PALMEIRA; MACHADO, 2011; MULLER et al., 2008).

No que diz respeito à variável idade, foi observado que a maioria dos pacientes acometidos encontrava-se na faixa etária entre 50 a 69 anos, em estudos com resultados semelhantes, os pacientes com diagnóstico de IAM apresentaram idade média de 60 anos (MATTOS et al., 2002; MUNHOZ; OLIVEIRA, 2000; ESCOSTEGUY et al., 2003; BORDON et al., 2004; SOUSA et al., 2006; EVANGELISTA; BARRETO; GUERRA, 2008).

Esta pesquisa demonstrou predominância quanto ao gênero masculino de $10(71,4 \%)$ pacientes, corroborando com a literatura (ESCOTEGUY et al., 2003). Muller e colaboradores (2008) realizaram uma pesquisa que demonstrou 146 pacientes com diagnóstico de IAM, sendo 94 pacientes homens, enquanto que apenas 52 pacientes eram mulheres. Informação também confirmada por Evangelista, Barreto e Guerra (2008), que realizaram uma pesquisa no Estado de Minas Gerais, demonstrando $57,8 \%$ dos pacientes internados por doenças isquêmicas do coração sendo do gênero masculino.

Em contradição aos achados acima, um estudo observou que dos pacientes admitidos em centros de tratamento de cardiologia em dois locais diferentes, a maioria pertencia ao gênero feminino (MUNHOZ; OLIVEIRA, 2000).

Em relação ao período de atendimento dos pacientes, entre noturno e diurno, 10 (71,4\%) pacientes deram entrada no Pronto Atendimento (PA) antes das 18 horas, e 4 $(28,6 \%)$ após esse horário. Um estudo realizado por Muller e Colaboradores (2008) apresentou situação semelhante, em que existia uma diminuição de atendimentos no período noturno.

Referente ao tempo de apresentação da dor precordial, observou-se que em 6 $(42,9 \%)$ pacientes não houve registro do dado na ficha de atendimento, fato que demonstra uma deficiência no registro das informações. Quanto aos demais dados encontrados, observou-se que $3(21,4 \%)$ pacientes relataram 0 início da dor precordial entre 1 e 2 horas e 4 (28,6\%) entre 2 e 4 horas, enquanto que apenas 1 $(7,1 \%)$ relatou início da dor precordial há mais de 12 horas. Em estudo apresentado em 2004, por Bordon e colaboradores, indicou que o tempo de dor precordial, do início dos sintomas até o momento da primeira avaliação foi relativamente longo. Entretanto, na pesquisa de Escosteguy e colaboradores (2003), realizada em pacientes atendidos pelo SUS, evidenciouse que 0 início dos sintomas até a hospitalização foi de até 6 horas.

Quanto à variável relacionada ao tempo porta-eletrocardiograma, vale ressaltar que, em comparação com o tempo ideal para realização do exame, os dados 
encontrados demonstraram que o exame foi mais demorado. Todavia, das 14 fichas de pacientes analisadas, observou-se a anotação do tempo porta-eletrocardiograma em 13 fichas, em que apenas $3(21,4 \%)$ dos pacientes foram submetidos ao eletrocardiograma nos primeiros 10 minutos, a partir do horário de abertura da ficha, enquanto que $10(71,4 \%)$ aguardaram um período superior ao adequado para realização do referido exame.

Segundo a recomendação da I Diretriz da Dor Torácica (2002), todo paciente, com suspeita de SCA na sala de emergência, deve realizar imediatamente um ECG, em 5 a 10 minutos (BASSAN et al., 2002). De acordo com dados estatísticos, 13 pacientes tiveram média de 26,6 minutos de espera, $(P=0,015)$. Esse dado vem de encontro a uma pesquisa realizada em 2008, por Muller e colaboradores, que a média apresentada, no tempo porta-eletrocardiograma, foi de 19,4 minutos, ocasionando, inclusive, a demora para a administração da terapia trombolítica.

Conforme a presente pesquisa, 6 $(42,9 \%)$ das fichas consultadas apresentaram alteração no ECG, 3 (21,4\%) apresentaram 0 exame nos padrões considerados normais, e 5 (35,7\%) fichas não continham o exame anexado e algum registro dessa informação, fato que demonstra a deficiência no registro de um dado importante referente ao atendimento prestado. O estudo realizado por Muller e colaboradores (2008) demonstrou que houve alteração do ECG em $91,6 \%$ dos casos.

Ressalta-se a importância do Enfermeiro em reconhecer os sintomas do IAM com a finalidade de agilizar o atendimento, priorizando a realização do ECG no menor tempo possível. Por essas razões, necessita-se avaliar a situação dos atendimentos, e investigar possíveis fatores que interferiram no tempo gasto para que o paciente seja atendido no menor tempo possível (PALMEIRA; MACHADO, 2011; PIERCE et al., 2009; SAVONITTO et al., 1999).

As Unidades de PA são responsáveis por tratamentos especializados que devem ser de forma sistemática, otimizando recursos para garantir o atendimento seguro e de qualidade para os pacientes.

\section{CONCLUSÃO}

Os dados obtidos no presente estudo indicam que houve uma média de 26,6 minutos para a realização do ECG que contradiz o protocolo estabelecido pela I Diretriz de Dor Torácica em Sala de Emergência, tempo de 10 minutos para a realização desse importante exame.

\section{REFERÊNCIAS}

AFILALO, J.; ROY, A. M.; EISENBERG, M. J. Systematic review of fibrinolytic-facilitated percutaneous coronary intervention: Potential benefits and future challenges. Can. J. Cardiol, Montreal, v. 25, n. 3, p. 141148, Mar. 2009.

BASSAN, R. et al. Sociedade Brasileira de Cardiologia. I Diretriz de dor torácica na sala de emergência. Arq. Bras. Cardiol., São Paulo, v. 79, n. 2, p. 1- 22, 2002.

BORDON, J. G. et al. Redução da mortalidade após implementação de condutas consensuais em pacientes com infarto agudo do miocárdio. Arq. Bras. Cardiol., Botucatu, v. 82 , n. 4, p. $370-373$, abr. 2004 
ESCOSTEGUY, C. C. et al. Infarto agudo do miocárdio: perfil clínico-epidemiológico e fatores associados ao óbito hospitalar no município do Rio de Janeiro. Arq. Bras. Cardiol., Rio de Janeiro, V. 80, n. 6, p. 593599, 2003.

EVANGELISTA, P. A.; BARRETO, S. M.; GUERRA, H. L. Central de regulação de leitos do SUS em Belo Horizonte, Minas Gerais, Brasil: avaliação de seu papel pelo estudo das internações por doenças isquêmicas do coração. Cad Saúde Pública,

Rio de Janeiro, v. 24, n. 4, p. 767-776, abr. 2008.

MARINO, P. L. Compêndio de UTI. 2. ed. Porto Alegre: Artes Médicas Sul, 1999.

MATTOS, L. A. et al. Evolução temporal com a utilização da angioplastia coronariana primária no infarto agudo do miocárdio no Brasil. Análise dos preditores de sucesso e dos eventos adversos hospitalares em 9.434 pacientes. Arq. Bras. Cardiol., São Paulo, v. 79, n. 4, p. 405-411, set. 2002.

MULLER, L. A. et al. Fatores que retardam a administração de trombolitico em pacientes com diagnóstico de infarto agudo do miocárdio atendidos em um hospital geral. Rev Latino-am. Enfermagem, Ribeirão Preto, v. 16, n. 1, p. 52-56, jan-fev. 2008.

MUNHOZ, E. C.; OLIVEIRA, P. F. Significado Clínico da Reoclusão Hospitalar no Infarto Agudo do Miocárdio tratado com reperfusão mecânica e angioplastia coronariana primária. Arq. Bras. Cardiol., Curitiba, v. 75, n. 5, p. 429-435, 2000.
MUSSI, F. C. O infarto e a ruptura com o cotidiano: possível atuação da enfermagem na prevenção. Rev. Latino-am. Enfermagem, Ribeirão Preto, v. 12, n. 5, p. 751-759, setout. 2004.

PALMEIRA, N. C. L.; MACHADO, R. C. Tempo porta eletrocardiograma: avaliação do atendimento a pacientes com infarto agudo do miocárdio. Rev enferm UFPE on line, Recife, v. 5, n. 8, p. 1898-1904, out. 2011.

PIEGAS L. S. et al. IV Guideline of the Brazilian Society of Cardiology Treatment of Acute Myocardial Infarction with ST-segment elevation. Arq Bras Cardiol, São Paulo, v. 93, n. 6, p. 179-264, 2009.

PIERCE, J. D. et al. Heart Failure Guidelines and Implications for Surgically Treating Heart Failure. AORN J, Kansas, v. 90, n. 6, p. 874888, dez. 2009.

SAVONITTO, S. et al. Prognostic value of the admission electrocardiogram in acute coronary syndromes. JAMA, Chicago, v. 281, n. 8, p. 707-713, fev. 1999.

SILVEIRA, F. R. Infarto agudo do miocárdio - tratamento trombolítico. In: DARWICH, R. N. Condutas e Rotinas em Terapia Intensiva. Rio de Janeiro: Revinter, 2002. p. 17-19.

SOUSA, J. M. A. et al. Comparação da coronariografia de mulheres diabéticas e não-diabéticas com síndrome coronariana aguda sem supradesnivelamento de ST. Arq. Bras. Cardiol., São Paulo, v. 86, n. 2, p. 150155, fev. 2006. 